\title{
NEUROPATIA PUDENDA:
}

\section{correlação com dados demográficos, índice de gravidade e parâmetros pressóricos em pacientes com incontinência fecal}

\author{
Luciano Pinto de CARVALHO ${ }^{1}$, Oly Campos CORLETA ${ }^{2}$, \\ Afonso Calil Mury MALLMANN ${ }^{1}$, Ruy Takashi KOSHIMIZU ${ }^{1}$ e \\ Alessandro SPOLAVORI ${ }^{3}$
}

RESUMO - Racional - A incontinência feccal é a incapacidade de manter o controle da eliminação do conteúdo intestinal em local e tempo socialmente adequados, resultando em escape de gases e fezes. Sua investigação diagnóstica exige múltiplos exames. A manometria anorretal é considerada imprescindível nesta avaliação. A correlação da manometria com estudos eletrofisiológicos e os sintomas ainda não estão completamente estabelecidos na literatura. Objetivos - Correlacionar os dados da manometria anorretal e o estudo do tempo de latência motora terminal do nervo pudendo em pacientes com incontinência fecal e comorbidades. Pacientes e Métodos - Foram estudados prospectivamente 39 pacientes, 85,6\% do sexo feminino com idade média de 60,1 anos $( \pm 12,89)$, com queixa clínica de incontinência fecal atendidos em ambulatório entre março de 1997 e junho de 2000 no Laboratório de Fisiologia Anorretal do Serviço de Coloproctologia do Hospital Nossa Senhora da Conceição, Porto Alegre, RS. Foram realizados índice de incontinência, manometria anorretal, estudo do tempo de latência motora terminal do nervo pudendo bilateralmente e exame proctológico em todos os pacientes. Para análise estatística, os pacientes foram separados em grupos segundo resultados da manometria anorretal, neuropatia pudenda, idade e sexo. Resultados/Conclusão - A média do índice de incontinência fecal dos pacientes estudados foi de 9,30 \pm 4,93. À manometria anorretal, 23 pacientes (59\%) apresentaram pressões reduzidas. As pressões foram significativamente mais elevadas nos pacientes do sexo masculino. O tempo de latência motora terminal do nervo pudendo (neuropatia) foi prolongado em 14 doentes $(35,9 \%)$. A idade e o tempo de latência motora terminal do nervo pudendo correlacionaram-se significativamente. A demais correlações não foram estatisticamente significativas.

DESCRITORES - Incontinência fecal, etiologia. Manometria. Ânus, fisiologia. Reto, fisiologia. Defecação, fisiologia. Eletrofisiologia.

\section{INTRODUÇÃO}

Incontinência fecal é a incapacidade de manter o controle fisiológico do conteúdo intestinal em local e tempo socialmente adequados. Os sintomas podem variar do escape ocasional de flatos, até a perda contínua involuntária de fezes.

A real prevalência da incontinência fecal na população é de difícil determinação. Estudos em populações distintas mostraram prevalência entre $0,1 \%$ a $15 \%^{(3,5,12,20,40,41)}$. Na população brasileira, estudo realizado em ambulatório de geriatria no Hospital de Clínicas de São Paulo, encontrou prevalência de $10,9 \%{ }^{(14)}$.

A avaliação do grau de incontinência atualmente inclui escalas que utilizam índices de pontuação para o tipo de escape, sua freqüência, relação com a qualidade de vida, seu impacto na vida dos pacientes, avaliando limitações físicas e sociais, como a necessidade do uso de forro perineal $^{(7,21,29)}$.

\footnotetext{
Trabalho apresentado como dissertação de mestrado na Universidade Federal do Rio Grande do Sul, para obtenção do título de mestre em Medicina.

${ }^{1}$ Serviço de Coloproctologia do Hospital Nossa Senhora da Conceição, Porto Alegre, RS; Departamento de Cirurgia da Universidade Federal do Rio Grande do Sul, Porto Alegre, RS
}

Endereço para correspondência: Dr. Luciano Pinto de Carvalho - Rua Amélia Telles, 262 - apto. 401 - Petrópolis - 90460-070 - Porto Alegre, RS. e-mail: lucianocarvalho@terra.com.br 
A manutenção da continência fecal é dependente de vários mecanismos anatômicos e fisiológicos interrelacionados, como a integridade do complexo esfincteriano anal, integridade do assoalho pélvico, capacidade e complacência retal, assim como sensação anorretal, consistência e volume das fezes, tempo de trânsito colônico, que necessitam interagir para controlar a fisiológica manutenção e eliminação do conteúdo intestinal ${ }^{(24,33)}$. As causas mais comuns são a traumática e a idiopática ${ }^{(31)}$. Outro subgrupo classificado como de etiologia idiopática, no qual os pacientes não apresentam nenhuma evidência de anormalidade anorretal, pode estar associado à neuropatia do assoalho pélvico com aumento do tempo de latência motora do nervo pudendo ${ }^{(11)}$.

Os exames disponíveis para investigação fisiológica do ânus e reto tiveram início com a manometria anorretal ${ }^{(6)}$ e hoje inclui uma série de exames que são complementares para a integral avaliação fisiológica da incontinência fecal ${ }^{(9,37)}$. A associação de neuropatia de nervo pudendo com incontinência fecal e a correlação com gravidade dos sintomas, dados manométricos e seu valor prognóstico foram objeto de muitos estudos na última década, com resultados conflitantes $^{(23,25)}$.

O objetivo deste estudo foi avaliar a correlação entre os dados da manometria anorretal e o tempo de latência motora terminal do nervo pudendo em pacientes com incontinência fecal. Propôs-se também conhecer o grau de incontinência fecal e a sua correlação com os dados da manometria anorretal e o tempo de latência motora terminal do nervo pudendo, assim como correlacionar esses dados com as comorbidades em pacientes com incontinência fecal.

\section{PACIENTES E MÉTODOS}

\section{Delineamento e Métodos}

Este é um estudo transversal, em que foram estudados 39 pacientes atendidos no Laboratório de Fisiologia Anorretal do Serviço de Coloproctologia do Hospital Nossa Senhora da Conceição, Porto Alegre, RS, com queixa clínica de incontinência fecal no período de março de 1997 até junho de 2000. Os critérios de exclusão foram os pacientes que não permitiram a realização de manometria anorretal ou tempo de latência motora terminal do nervo pudendo, com cirurgias colorretais baixas prévias, com tumores anorretais e idade abaixo de 12 anos. Todos os pacientes elegíveis para o estudo foram submetidos a aplicação de questionário contendo informações quanto à idade, sexo, características da incontinência e história médica pregressa, manometria anorretal, exame físico e proctológico, estudo da latência motora terminal do nervo pudendo bilateralmente. Para classificar clinicamente o grau de incontinência fecal, foi utilizado o índice de incontinência proposto por JORGE e WEXNER(7).

\section{Manometria Anorretal}

O sistema de manometria anorretal utilizado foi de microbalão de água, marca Proctosystem PL3000, sendo a unidade de medida em centímetros de água. As medidas foram obtidas com intervalos de $1 \mathrm{~cm}$ (pontos), utilizando-se a técnica estacionária a cada ponto, com tração manual, iniciando-se no reto a $6 \mathrm{~cm}$ da margem anal, progredindo para o canal anal até $1 \mathrm{~cm}$. As medidas obtidas foram as seguintes: pressão de repouso (PR), pressão de contração voluntária (PCV), medida do comprimento do canal anal e reflexo inibidor retoanal (RIRA). Os parâmetros de normalidade considerados foram de 40 a $80 \mathrm{~cm} \mathrm{H}_{2} \mathrm{O}$ para pressão de repouso, e de 80 a 180 cm $\mathrm{H}_{2} \mathrm{O}$ para pressão de contração voluntária. Os parâmetros de normalidade foram os de estudo piloto realizado no Laboratório de Fisiologia Anorretal do Hospital Nossa Senhora da Conceição, em indivíduos sem incontinência fecal. Foram considerados como tendo manometria alterada os pacientes com pressões de repouso ou de contração voluntária abaixo dos valores normais.

\section{Testes de Sensibilidade Retal}

Os testes de sensibilidade retal foram realizados com o balão intra-retal. Os valores considerados normais foram de 20 a $60 \mathrm{~mL}$ de ar para o limiar, e de 60 a $180 \mathrm{~mL}$ de ar para o volume máximo ${ }^{(9)}$.

\section{Exame Proctológico}

O exame proctológico foi executado após a realização da manometria anorretal, e incluiu inspeção da região anal, palpação, toque retal e retossigmoidoscopia rígida. No toque retal foram considerados como defeito esfincteriano, falha ou zona de fibrose com defeito na musculatura esfincteriana anal, perceptível e identificado pelo examinador. Os pacientes foram classificados em: com incontinência traumática, quando apresentavam defeito esfincteriano ao toque, e com incontinência idiopática, quando não apresentavam defeito ou outra causa específica identificada.

\section{Latência Motora Terminal do Nervo Pudendo}

O estudo do tempo de latência motora terminal do nervo pudendo foi medido bilateralmente, com o paciente em decúbito lateral esquerdo. $\mathrm{O}$ método utilizado foi o descrito por KIFF e SWASH ${ }^{(10)}$ (eletrodo de St Mark's Pudendal Electrode-Dantec 90130240, Skovlunde, Dinamarca). O eletrodo era conectado a um aparelho de eletromiografia (marca Dantec - Cantata), sendo considerados normais os valores até 2,0 $\pm 0,2$ milisegundos $^{(43)}$ (Figura 1). A presença de neuropatia de nervo pudendo foi considerada quando os valores do tempo de latência motora terminal deste foram acima de 2,2 milisegundos, uni e ou bilateralmente. 


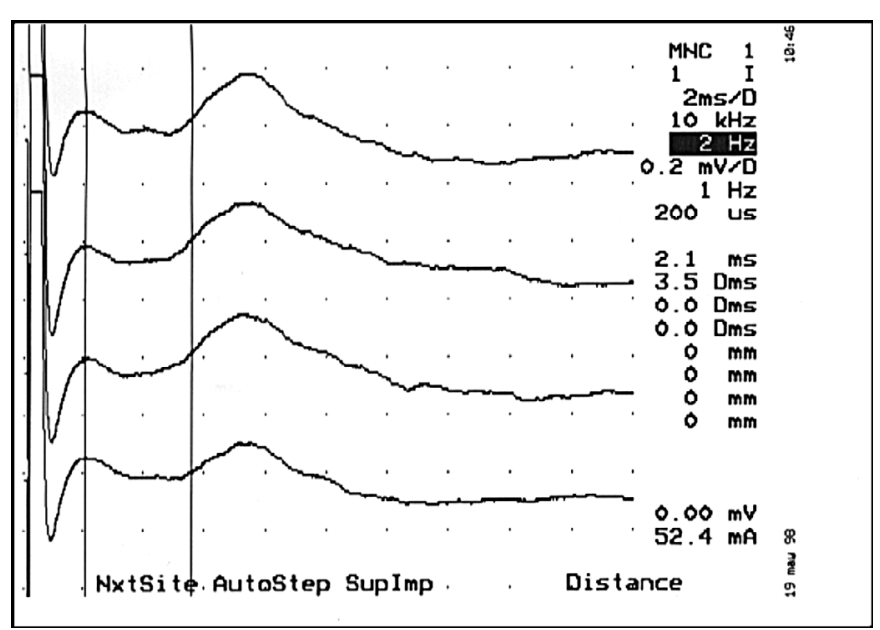

FIGURA 1 - Registro do tempo de latência motora terminal do nervo pudendo

\section{Considerações Éticas}

Este trabalho foi submetido a apreciação e aprovado pelo Grupo de Pesquisa e Pós-Graduação do Hospital de Clínicas de Porto Alegre e pela Comissão de Ética do Centro de Aperfeiçoamento e Pesquisa do Hospital Nossa Senhora da Conceição.

Os pacientes foram informados quanto aos aspectos éticos desta pesquisa através de termo de consentimento informado.

\section{Análise Estatística}

Inicialmente, foram obtidas tabelas de freqüência para todas as variáveis do banco de dados. As variáveis quantitativas foram descritas através de média e desvio padrão e as variáveis categóricas através de percentuais. Para efeito de comparação, os pacientes foram analisados de maneira global e divididos em grupos (manometria alterada e manometria normal; neuropatia de pudendo e sem neuropatia; idade abaixo de 60 anos e a partir de 60; sexo masculino e feminino). As comparações de variáveis quantitativas entre grupos dicotômicos foram feitas pelo $t$ de Student. A avaliação da associação entre duas ou mais variáveis quantitativas foi feita através do coeficiente de correlação linear de Pearson. A confirmação de associação entre variáveis categóricas foi feita pelo teste de Qui-quadrado. O nível de significância considerada foi de alfa $=0,05$. Os dados foram analisados e processados com auxílio dos programas Epi-info e SPSS for Windows.

\section{RESULTADOS}

Foram estudados 39 pacientes: 6 do sexo masculino $(15,4 \%)$ e 33 do sexo feminino $(84,6 \%)$. Na manometria anorretal, a média das pressões de repouso obtidas foram de $50,18 \pm 20,87$, com valores abaixo do normal em 22 doentes (56,40\%). A média das pressões de contração voluntária foi de $90,13 \pm 39,90 \mathrm{~cm} \mathrm{H_{2 }} \mathrm{O}$, estando diminuídas em 23 doentes $(59,0 \%)$. Sensibilidade retal alterada foi encontrada em 13 doentes $(34,2 \%)$.

O tempo de latência motora terminal do nervo pudendo foi em média de 2,24 $\pm 0,60$ milisegundos no lado direito e de 2,09 $\pm 0,55$ milisegundos no esquerdo. Neuropatia de nervo pudendo foi encontrada em 14 (35,9\%) dos pacientes estudados. As características gerais da amostra encontram-se na Tabela 1. A incontinência foi idiopática em 28 pacientes (71,8\%), traumática em $10(10,2 \%)$ e neurológica, por trauma raquimedular, em 1 doente (2,6\%).

As correlações entre pressão de repouso, pressão de contração voluntária, índice de incontinência e tempo latência de nervo pudendo direito e esquerdo mostraram-se fracas, sem significância estatística, como demonstrado na Tabela 2. As correlações entre pressão de repouso e de contração voluntária com o tempo de latência motora terminal de nervo pudendo, usando-se a medida bilateral mais alta, não foram significativas.

As correlações do número de partos com diminuição da pressão de repouso $(P=0,191)$, com pressão de contração voluntária $(P=0,109)$, com o índice de incontinência $(P=0,130)$ e com o tempo de latência do nervo pudendo $(P=0,246)$ não foram estatisticamente significativas.

TABELA 1 - Características gerais dos pacientes estudados $(\mathrm{n}=39)$

\section{CARACTERÍSTICA}

Idade, anos

$60,1 \pm 12,9$

Sexo feminino, $\mathrm{f}(\%)$

$33(84,6)$

Sexo masculino, $\mathrm{f}(\%)$

$6(15,4)$

Início dos sintomas, anos

$5,57 \pm 7,68$

Índice de incontinência

$9,30 \pm 4,93$

Partos p/paciente $(n=33)$

$3,42 \pm 3,61$

Manometria anorretal

pressão de repouso, $\mathrm{cm} \mathrm{H}_{2} \mathrm{O}$

$50,18 \pm 20,87$

pressão contração voluntária, cm $\mathrm{H}_{2} \mathrm{O}$

$90,13 \pm 39,90$

comprimento canal anal, $\mathrm{cm}$

$2,71 \pm 1,05$

reflexo inibidor retoanal presente, $\mathrm{f}(\%)$

$35(89,7)$

Sensibilidade retal

limiar, $\mathrm{mL}$ de ar

$54,49 \pm 33,0$

volume máximo tolerado, $\mathrm{mL}$ de ar

$163,97 \pm 71,39$

Toque retal

$$
\text { com defeito esfincteriano, } \mathrm{f}(\%)
$$$$
10(25,64)
$$$$
\text { sem defeito esfincteriano, } \mathrm{f}(\%)
$$

Tempo de latência motora nervo pudendo, ms direito

$2,24 \pm 0,60$

esquerdo

$2,09 \pm 0,55$

Neuropatia uni ou bilateral, $f(\%)$

$14(35,9)$

$\mathrm{ms}=$ milisegundos 
TABELA 2 - Coeficiente de correlação e significância estatística das correlações entre idade, pressão de repouso, pressão de contração voluntária, tempo de latência de nervo pudendo direito e esquerdo e índice de incontinência

\begin{tabular}{|c|c|c|c|c|c|}
\hline & $\begin{array}{l}\text { Pressão de } \\
\text { repouso }\end{array}$ & $\begin{array}{c}\text { Pressão } \\
\text { contração } \\
\text { voluntária }\end{array}$ & $\begin{array}{l}\text { Tempo de } \\
\text { latência } \\
\text { direito }\end{array}$ & $\begin{array}{l}\text { Tempo de } \\
\text { latência } \\
\text { esquerdo }\end{array}$ & $\begin{array}{c}\text { Índice } \\
\text { incontinência }\end{array}$ \\
\hline Idade & $P=0,177$ & $P=0,597$ & $P=0,011$ & $P=0,017$ & $P=0,229$ \\
\hline Pressão de repouso & & $r=0,616$ & $r=-0,226$ & $r=-0,040$ & $\mathrm{r}=-0,035$ \\
\hline Pressão contração voluntária & & & $P=0,535$ & $P=0,692$ & $P=0,605$ \\
\hline \multirow[t]{2}{*}{ Tempo de latência direito } & & & & $\mathrm{r}=0,723$ & $\mathrm{r}=0,184$ \\
\hline & & & & $P<0,001$ & $P=0,260$ \\
\hline \multirow[t]{2}{*}{ Tempo de latência esquerdo } & & - & - & 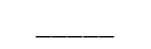 & $\mathrm{r}=0,184$ \\
\hline & & & & & $P=0,260$ \\
\hline
\end{tabular}

Houve correlação forte entre a idade com o tempo de latência motora terminal de nervo pudendo, considerando-se o valor mais alto da medida bilateralmente, $\mathrm{r}=0,422(P=0,007)$. Para cada 10 anos de idade, o tempo de latência de nervo pudendo aumentou aproximadamente 0,2 milisegundos (Figura 2). As demais correlações com a idade foram fracas, sem significância estatística.
A comparação entre grupos com e sem neuropatia de nervo pudendo demonstrou a presença desta última em 14 doentes. O grupo com neuropatia teve média de idade superior, maior número de mulheres, o índice de incontinência mais alto e pressões de repouso na manometria mais baixas, porém estes achados não tiveram significância estatística (Tabela 3).

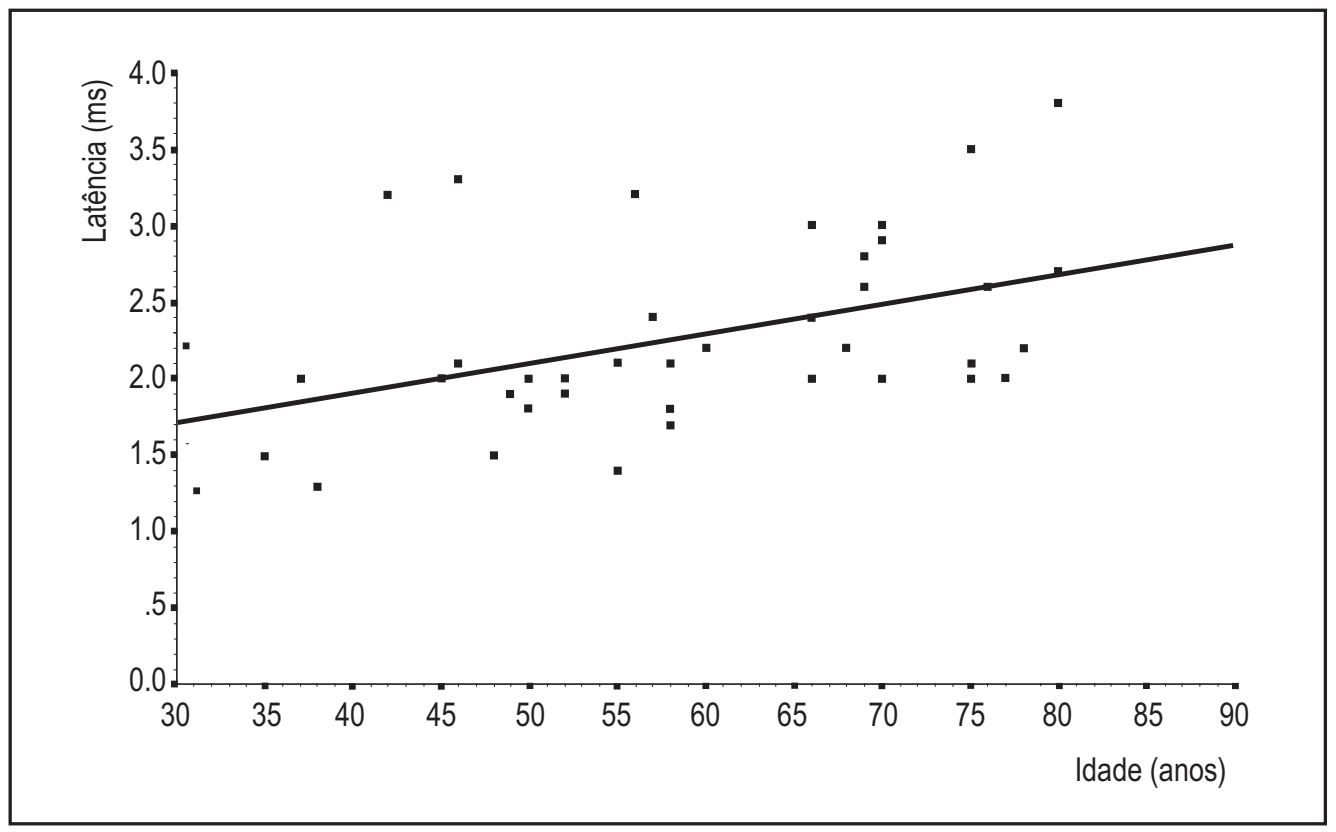

FIGURA 2 - Gráfico de dispersão de pontos representando a correlação entre idade e latência motora terminal de nervo pudendo (medida do valor maior bilateral), $\mathrm{r}=0,422(P=0,007)$. Para cada 10 anos de idade, a latência do nervo pudendo aumentou em aproximadamente $0,2 \mathrm{~ms}$ 
Carvalho LP, Corleta OC, Mallmann ACM, Koshimizu RT, Spolavori A. Neuropatia pudenda: correlação com dados demográficos, índice de gravidade e parâmetros pressóricos em pacientes com incontinência fecal

TABELA 3 - Comparação entre grupos de acordo com o estudo da latência motora terminal do nervo pudendo

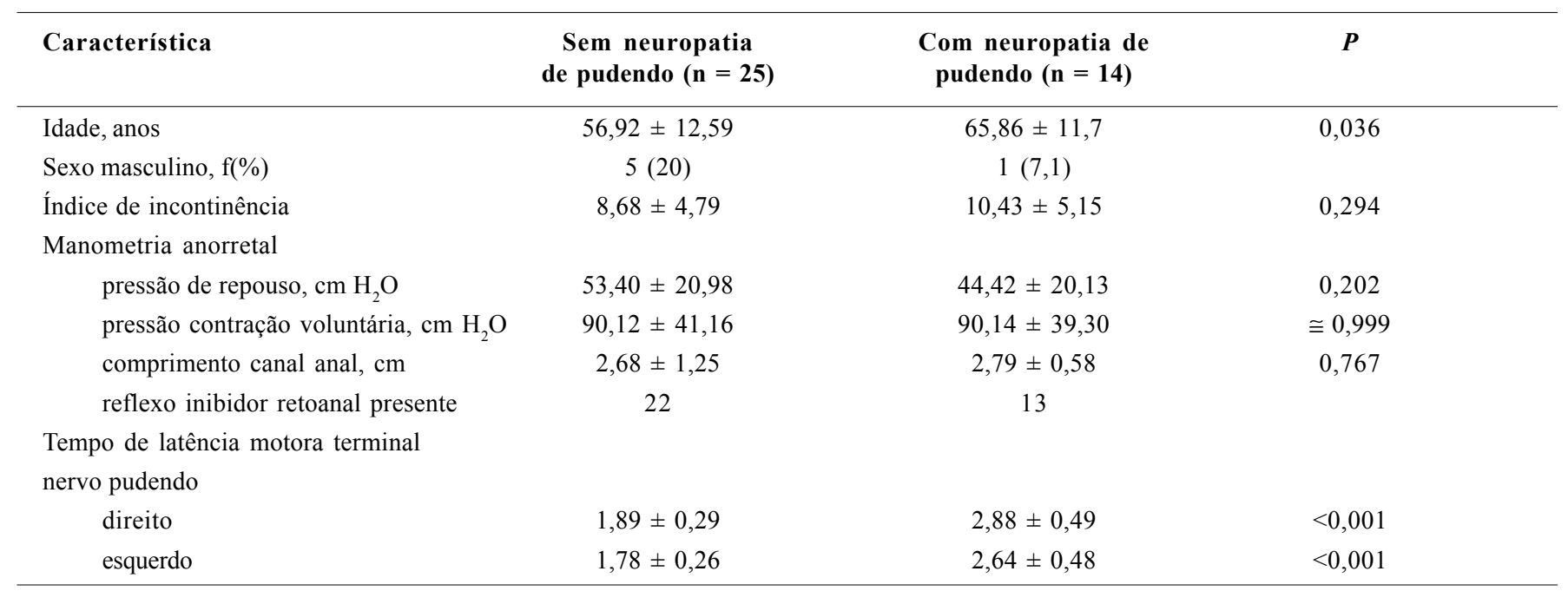

Comparação entre grupos com manometria normal e manometria alterada mostrou-se sem diferenças estatisticamente significativas. A comparação entre os grupos separados por idade, abaixo e acima de 60 anos, mostrou que o tempo de latência motora terminal do nervo pudendo em ambos os lados foi significativamente maior no grupo acima de 60 anos. Quando a comparação foi entre grupos separados por sexo, as médias das pressões de repouso e de contração voluntária no sexo masculino foram mais elevadas do que no sexo feminino, assim como o canal anal foi mais longo no sexo masculino, sendo estatisticamente significativos $(P<0,001)$.

\section{DISCUSSÃO}

A incontinência fecal é situação incapacitante com grandes repercussões socioeconômicas. Apesar de ser condição benigna, as alterações na qualidade de vida dos doentes justificariam o interesse maior da comunidade médica e da sociedade em tratar do assunto. As questões culturais que envolvem as doenças anorretais fazem com que sua real importância seja subestimada.

A freqüência exata da incontinência fecal permanece desconhecida. A prevalência varia de $0,1 \%$ a $15 \%$ em diversos estudos ${ }^{(3,5,12,20,40,41)}$.

A idade dos pacientes deste estudo variou de 35 a 80 anos, com média de $60,1 \pm 12,9$ anos. O sexo feminino foi predominante com $84,6 \%$ dos pacientes. Os dados são semelhantes aos da literatura, que demonstram que a prevalência da incontinência fecal é maior em mulheres $^{(19,20)} \mathrm{e}$ aumenta proporcionalmente à idade ${ }^{(16,35)}$.

$\mathrm{Na}$ presente série, a incontinência fecal foi classificada como idiopática na maioria dos pacientes estudados $(71,8 \%)$. Segundo a literatura, a incontinência é classificada como idiopática em aproximadamente $80 \%$ dos pacientes ${ }^{(32)}$. A definição e a incidência de incontinência idiopática precisam ser analisadas com maior atenção. A introdução do uso da ecografia endoanal tem mostrado existência de defeitos esfincterianos em mais de $30 \%$ de pacientes com incontinência dita idiopática ${ }^{(32)}$.

O parto vaginal com esforço excessivo pode ser fator de risco para estiramento de nervo pudendo, levando à neuropatia ${ }^{(36,39)}$. Comparações entre partos vaginais e partos cesáreos revelam que os primeiros estão associados com aumento no tempo de latência motora dos nervos pudendos, como demonstrado em $42 \%$ da série de pacientes de SNOOKS et al. ${ }^{(34)}$. No presente estudo, o número de partos por paciente não foi significativamente associado com o índice de incontinência. Porém, a média do número de partos foi superior no grupo com manometria alterada e no grupo com neuropatia de pudendo.

A avaliação do grau de incontinência tem importância tanto como parâmetro clínico, como para comparações futuras. A utilização de escalas com sistemas de índices que incluem também a repercussão na qualidade de vida dos doentes, é descrita por diversos autores ${ }^{(21)}$. Em estudo onde se compararam quatro escalas diferentes em diversos centros, houve forte correlação entre três de quatro escalas que também incluem a qualidade de vida na avaliação ${ }^{(28)}$. Apesar de não haver uma sistemática ideal, a uniformização da classificação permitiria a comparação entre os estudos sobre incontinência fecal.

O sistema utilizado neste estudo foi o proposto por JORGE e WEXNER $^{(7)}$, com pontuação de 0 para continência total e 20 para o pior grau de incontinência. No presente estudo, o índice variou de $4 \mathrm{a}$ 18 com média de 9,30 $\pm 4,93$. As comparações entre as médias dos índices nos grupos estudados não demonstraram diferenças 
estatisticamente significativas. As correlações entre índice de incontinência com idade, pressões da manometria, tempo de latência motora terminal de pudendo também não foram estatisticamente significativas (Tabela 2). A expectativa de que os pacientes com hipotonia à manometria anorretal apresentassem índices de incontinência mais elevados não foi confirmada na presente série. A principal razão para isto é que o índice valoriza muito a repercussão da incontinência na qualidade de vida dos pacientes, pontuando o uso de forros e as limitações físicas e sociais. Um paciente com incontinência para gases de ocorrência diária pode julgar que a repercussão dos seus sintomas é mais grave do que outro paciente com escape eventual de fezes sólidas, o que eleva o índice do primeiro. Este sistema de índice serve como parâmetro de comparação de tratamentos ou acompanhamento da gravidade dos sintomas.

No presente estudo, as correlações entre as pressões da manometria com idade e índice de incontinência não foram significativas. Em relação com a idade, a correlação das pressões da manometria foram fracas. A influência da idade nas medidas da manometria foi demonstrada em estudo de pacientes submetidos a avaliação fisiológica na Cleveland Clinic, Florida, onde forte correlação foi identificada em ambos os sexos, em que as pressões de repouso e de contração voluntária diminuem com a idade. Nas mulheres, esta redução se inicia em fase mais jovem; além disso, as pressões de repouso e de contração foram menores no grupo feminino comparado com o masculino ${ }^{(22)}$.

ENCK et al. ${ }^{(4)}$ estudaram 75 pacientes incontinentes, com média de idade de 48,7 $\pm 2,2$ anos, e encontraram forte correlação entre idade e pressão de repouso $(\mathrm{r}=-0,26, P=0,021)$. Além disso, as pressões no grupo feminino foram mais baixas que no grupo masculino.

Neste estudo, as pressões na manometria anorretal estavam abaixo do normal em 23 doentes (59\%), sendo este achado mais freqüente no sexo feminino do que no masculino $(P=0,033)$. As comparações entre os grupos estudados demonstraram que as pressões de repouso estavam mais baixas no grupo com neuropatia de pudendo e nos pacientes com mais de 60 anos, mas esta diferença não teve significância estatística. As pressões de contração foram semelhantes nos dois grupos separados por idade e presença de neuropatia de pudendo. As pressões de contração voluntária foram significativamente menores no sexo feminino $(P<0,001)$, assim como o canal anal foi mais curto nas mulheres $(P<0,001)$. Em outros estudos a avaliação manométrica em pacientes incontinentes demonstraram que as pressões de repouso e de contração são mais baixas e o canal anal funcional é mais curto, quando comparados com pessoas normais ${ }^{(2,8)}$.

A necessidade da interação das estruturas envolvidas no mecanismo esfincteriano é fundamental na manutenção da continência fecal. O fato de pacientes incontinentes apresentarem pressões normais da musculatura anal, demonstradas pela manometria, confirma que somente a medida do tônus não é capaz de diagnosticar a efetividade da função. Muitos pacientes apresentam tônus e força de contração normais, mas não conseguem manter esta força por muito tempo, o que pode ser a causa da incontinência. Alteração na sensibilidade retal em pacientes incontinentes foi encontrada por diversos autores ${ }^{(1,15}$ ${ }^{30)}$. Esta é mais freqüente nos pacientes diabéticos ${ }^{(1)}$. Outros estudos não encontraram alterações significativas ${ }^{(17,18)}$

A sensibilidade retal estava alterada em 13 (34,2\%) doentes no presente estudo. As comparações entre os grupos não mostraram diferenças significativas.

O método da medida do tempo de latência motora terminal do nervo pudendo foi desenvolvido por KIFF e SWASH ${ }^{(10)}$. A importância de tal estudo na incontinência fecal ainda é controverso na literatura. Também o complexo mecanismo da continência fecal ainda não é completamente esclarecido. Assim, a tentativa de atribuir causa única à incontinência, diagnosticada com um único exame é, no mínimo, muito simplista. O papel da inervação da musculatura do assoalho pélvico certamente é importante na manutenção do equilíbrio deste sistema. Afirmações de que alterações na velocidade de condução nervosa do nervo pudendo pode ser fator desencadeante da fraqueza da musculatura anal têm sido feitas em muitos estudos, com resultados controversos.

Os parâmetros normais da medida do tempo de latência motora terminal do nervo pudendo ainda constituem objeto de debate na literatura. Os valores acima dos quais o tempo de latência motora terminal do nervo pudendo é considerado prolongado variam de 2,1 milisegundos até 2,5 milisegundos, sendo considerados como diagnóstico de neuropatia do nervo valores superiores a esses ${ }^{(13,38,42)}$. No presente estudo adotou-se o valor de 2,2 milisegundos ${ }^{(43)}$. As diferenças entre os valores considerados normais tornam as comparações entre os estudos muito difíceis. A freqüência de neuropatia de pudendo variou, estando presente entre $21,1 \%$ e $44 \%$ dos pacientes incontinentes ${ }^{(23,26)}$. Outros estudos, no entanto, demonstraram que cerca de $80 \%$ das incontinências idiopáticas eram secundárias à neuropatia de pudendo ${ }^{(42,43)}$. A dificuldade de comparações entre os estudos está na diferença entre os grupos estudados e os parâmetros de normalidade adotados.

A presença de neuropatia de pudendo na presente série ocorreu em 14 doentes $(35,9 \%)$. O tempo médio de latência do nervo pudendo foi de 2,24 $\pm 0,60$ milisegundos no lado direito e 2,09 $\pm 0,55$ milisegundos no lado esquerdo. No grupo com idade acima de 60 anos, as medidas do tempo de latência foram maiores, estatisticamente significativas, em relação ao grupo com idade abaixo de 60 anos. A freqüência de neuropatia foi maior no grupo com manometria alterada, no grupo mais idoso e no das mulheres, mas sem significância estatística, o mesmo ocorrendo com as medidas do lado direito em comparação com as do lado esquerdo.

RIEGER et al. ${ }^{(27)}$ estudaram 67 pacientes ( 23 constipados e 44 incontinentes). Correlação negativa significativa foi encontrada entre a média do tempo de latência motora terminal do nervo pudendo e as 
pressões de repouso para pacientes incontinentes $(\mathrm{r}=-0,32, P=$ $0,037)$.

ROIG et al. ${ }^{(31)}$, estudando 44 pacientes com incontinência fecal e com idade média de 64 anos, encontraram freqüência de neuropatia em $37,2 \%$ dos pacientes. Nenhuma diferença significativa entre as pressões de repouso nos grupos com e sem neuropatia de pudendo foi demonstrada. A correlação entre tempo de latência de nervo pudendo e as pressões de repouso não foi significativa. Aumento no tempo de latência de nervo pudendo não implica necessariamente em pressões de repouso baixas.

No presente estudo as correlações entre pressões da manometria, tempo de latência de nervo pudendo e índice de incontinência foram fracas e sem significância estatística. A correlação se mostrou forte entre tempo de latência de nervo pudendo e idade, considerando-se o valor maior da medida bilateral da latência $(r=0,42, P=0,007)$. Para cada 10 anos de idade, o tempo de latência de nervo pudendo aumentou em aproximadamente 0,2 milisegundos nos pacientes incontinentes.

A complexidade do funcionamento das estruturas do assoalho pélvico ainda demanda estudos para o completo esclarecimento da manutenção da continência fecal. A escolha de um único exame para o diagnóstico e decisão terapêutica não deve ser rotina na investigação de pacientes com incontinência fecal. A manometria tem papel fundamental no arsenal diagnóstico, mas no presente estudo não foi demonstrada correlação significativa entre esta medida do tempo de latência motora terminal de nervo pudendo. Os pacientes com pressões manométricas baixas não irão necessariamente apresentar neuropatia de nervo pudendo.
A dificuldade de comparações entre os estudos na literatura justificaria a tentativa de uniformização dos parâmetros de normalidade. Também são necessários mais estudos para definir a real correlação entre os dados da manometria anorretal e a medida de latência motora terminal do nervo pudendo.

\section{CONCLUSÃO}

As correlações entre os dados da manometria anorretal e o tempo de latência motora terminal de nervo pudendo foram fracas e estatisticamente não-significativas, em pacientes com incontinência fecal.

Os pacientes com incontinência fecal apresentaram, em 59\% dos casos, pressões da manometria anorretal diminuídas, sendo que os do sexo masculino apresentaram canal anal mais longo e pressões de contração voluntária maiores em comparação com os do sexo feminino.

A prevalência de neuropatia de nervo pudendo foi de $35,9 \%$ entre pacientes com incontinência fecal avaliados

As correlações entre índice de incontinência, manometria, tempo de latência motora terminal de nervo pudendo e co-morbidades foram fracas, e estatisticamente não-significativas, nos pacientes com incontinência fecal.

A correlação entre idade e tempo de latência motora terminal de nervo pudendo foi forte, estatisticamente significativa: para cada 10 anos de idade, o tempo de latência de nervo pudendo aumentou 0,2 milisegundos.

Carvalho LP, Corleta OC, Mallmann ACM, Koshimizu RT, Spolavori A. Anorectal manometry and pudendal neuropathy association in fecal incontinence patients. Arq Gastroenterol 2002;39(3):139-146.

ABSTRACT - Background - Fecal incontinence stands for inability in maintaining the control of defecation to a socially acceptable and adequate time and place, resulting in unwanted release of gas, liquid or solid stool. The diagnosis needs multiple exams. Anorectal manometry is mandatory for this study. The correlation between manometry with electophysiological studies and symptoms is not yet clear in the literature. Aims - Correlate values of anal manometry, pudental nerve terminal motor latency and co morbidity in fecal incontinence patients. Methodology and Patients - Patients with clinical fecal incontinence, who attended the outpatient department of "Hospital Nossa Senhora da Conceição", Porto Alegre, RS, Brazil, between March 1997 and June 2000, were studied prospectively. Every single patient has undergone a general investigation, incontinence score, anal manometry, bilateral pudendal nerve terminal motor latency and physical examination. For statistical purposes the patients were separated in groups according to the results of the anal manometry, pudendal neuropathy, for age and sex. Results/ Conclusion - Thirty-nine patients were studied, $85,6 \%$ female, average age 60,1 years $( \pm 12,89)$. The average index of the fecal incontinence was of $9,30( \pm 4,93)$. In the anorectal manometry 23 patients showed low pressure. The pressures were significantly higher among males. The nerve terminal motor latency and pudental nerve (neuropathy) time was greater in 14 patients $(35,9 \%)$. The age and the time of pudental nerve terminal motor were significantly co related. The other co relations were not statistically significant.

HEADINGS - Fecal incontinence, etiology. Manometry. Anus, physiology. Rectum, physiology, Defecation, physiology. Electrophysiology. 


\section{REFERÊNCIAS BIBLIOGRÁFICAS}

1. Buser WD, Miner PB Jr. Delayed rectal sensation with fecal incontinence Gastroenterolgy 1986;91:1186-91.

2. Delechenaut P, Leroi AM, Weber J, Touchais JY, Czernichoww P, Denis P. Relatinoship between clinical symptoms of anal incontinence and the results of anorectal manometry. Dis Colon Rectum 1992:35:847-9.

3. Enck P, Bielefeldt K, Rathamann W, Purmann J, Tschüpe D, Erckenbrecht JF. Epidemiology of faecal incontinence in selected patients groups. Int J Colorectal Dis 1991;6:143-6.

4. Enck P, Kuhlbusch R, Lubke H, Frieling T, Erckenbrecht JF. Age and sex and anorectal manometry in incontinence. Dis Colon Rectum 1989;32:1026-30.

5. Giebel GD, Lefering R, Troidl H, Blöchl H. Prevalence of fecal incontinence: what can be expected? Int J Colorectal Dis 1998;13:73-7.

6. Gordon, Philip H, Nivatvongs S, editors. Principles and practice of surgery for the colon, rectum and anus. 2. ed. St. Louis, Missouri: Quality Medical Publishing; 1999. p.365-400.

7. Jorge JM, Wexner SD. Etiology and management of fecal incontinence. Dis Colon Rectum 1993;36:77-97

8. Jorge JMN, Wexner SD, Ehrenpreis, Nogueras JJ, Jagelman DG. Does perineal descent correlate with pudendal neuropathy? Dis Colon Rectum 1992;35:11-2.

9. Jorge JMN, Wexner SD. Anorectal manometry: techniques and clinical applications. South Med J 1993;86:924-31.

10. Kiff ES, Swash M. Slowed conduction in the pudendal nerves in idiopathic (neurogenic) faecal incontinence. Br J Surg 1984:71:614-6.

11. Kiff ES, Barnes PR, Swash M. Evidence of pudendal neuropathy in patients with perineal descene and chronic straining at stoll. Gut 1984;25:1279-82.

12. Groutz A, Fait G Lessing JB, David MP, Wolman I, Jaffa A, Gordon D. Incidence and obstetric risk factors of postpartum anal incontinence. Scand J Gastroenterol 1999:34:315-8.

13. Leroi A, Dorival M, Lecouturier CS. Pudendal neuropathy and severity of incontinence but not presence of an anal sphincter defect may determine the response to biofeedback therapy in fecal incontinence. Dis Colon Rectum 1999;42:762-9.

14. Lopes MC. Prevalência da incontinência anal no idoso. Estudo epidemiológico com base na população atendida no Hospital das Clínicas da Faculdade de Medicina da Universidade de São Paulo. Em regime ambulatorial (dissertação). São Paulo: Faculdade de Medicina da Universidade de São Paulo; 1994.

15. Lubowski DZ, Nicholls RJ. Faecal incontinence associated with reduced pelvic sensation. Br J Surg 1988;75:1086-8.

16. Mandelstam DA. Faecal incontinence. A social and economic factors. In: Henry MM, Swash M, editors. Coloproctology and the pelvic floor: pathophysiology and management. London: Butterwoth; 1984. p.217-22.

17. Miller R, Bartolo DCC, Cervero F, Moertensen NJ. Anorectal temperture sensation: a comparison of normal and incontinent patients. Br J Surg 1987;74:511-5.

18. Miller R, Bartolo DCC, Cervero F, Moertensen NJ. Anorectal sampling: a comparison of normal and incontinence patients. Br J Surg 1988;75:44-7.

19. Milne JS. Prevalence of incontinence in the elderly age group. In: Wilington EL, editor. Incontinence in elderly. London: Academic Press; 1976. p.9.

20. Nelson R, Norton N, Cauttley E, Furner S. Community-based prevalence of anal incontinence. JAMA 1995;274:559-61.

21. Pescatori M, Anastasio G, Bottini C, Mentasti A. A new grading and scoring for anal incontinence: evaluation of 335 patients. Dis Colon Rectum 1992;35:482-7.

22. Pfeifer J, Oliveira L, Park UC, Wexner SD. The relation of manometry to age and gender. Tech Coloproctol 1996;1:10-3.
23. Pfeifer J, Salanga VD, Agachan F, Weiss EG, Wexner SD. Variation in pudenda nerve terminal motor latency according to disease. Dis Colon Rectum 1997;40:79-83.

24. Phillips SF, Giller J. The contribution of the colon to the electrolyte and wate absortion in man. J Lab Clin Med 1973;81:733-46.

25. Rao SS, Patel RS. How useful are manometrical tests of anorectal function in the management of defecation disorders. Am J Gastroenterol 1997;92:469-75.

26. Rasmussen OO, Christiansen J, Tezschner T, Sorensen M. Pudendal nerve function in idiopathic fecal incontinence. Dis Colon Rectum 2000;43:633-6.

27. Rieger NA, Sarre RG, Saccone GT, Schloithe AC, Wattchow DA. Correlation of pudendal nerve terminal latency with the results of anal manometry. Int J Colorectal Dis 1997;12:303-7.

28. Rockwood TH, Church JM, Fleshman JW, Kane RL, Mavrantonis C, Thorson AG, Wexner SD, Bliss D, Lewry AL. Patient and surgeon ranking of the severity of symptoms associated with fecal incontinence. Dis Colon Rectum 1999;42:1525-32

29. Rockwood TH, Church JM, Fleshman JW, Kane RL, Mavrantonis C, Thorson AG, Wexner SD, Bliss D, Lewry AL. Fecal incontinence quality of life scale: quality of life instrument for patients with fecal incontinence. Dis Colon Rectum 2000;43:9-16; discussion 16-7.

30. Rogers J, Levy DM, Henry MM, Misiewicz JJ. Pelvic floor neuropathy: a comparative study of diabetes melittus and idiopathic faecal incontinence. Gut 1988;29:756-61.

31. Roig JV, Villoslada C, Lledó S, Solana A, Buch E, Alós R, Hinojosa J. Prevalence of pudendal neuropathy in fecal incontinence. Results of a prospective study. Dis Colon Rectum 1995;38:952-8.

32. Sagar PM, Pemberton JH. Anorectal and pelvic floor function. Gastroenterol Clin North Am 1996;25:163-82.

33. Schiller LR. Faecal incontinence. Clin Gastroenterol 1986;15:687-704.

34. Snooks SJ, Swash M, Stetchell, Henry MM. Injury to innervation of pelvic sphincter musculature in childbirth. Lancet 1984;2:546-50.

35. Snooks SJ, Henry MM, Swash M. Faecal incontinence due to external ana sphincter division in childbirth is associated to the innervation of the pelvic floor musculature: a double pathology. Br J Obstet Gynecol 1985;92:824-8.

36. Snooks SJ, Swash M, Henry MM, Setchell M. Risk factors in childbirth causing damage to the pelvic floor innervation. Br J Surg 1985;72:S15-7.

37. Swash M, Snooks SJ. Motor nerve conduction studies of the pelvic floo innervation. In: Henry MM, Swash M, editors. Coloproctology and the pelvic floor. 2.ed. London: Butterworth-Heinemann; 1992. p.196-206.

38. Swash M. Histopathology of the pelvic floor muscles. In: Henry MM, Swash M, editors. Coloproctology and the pelvic floor. London: Butterworths; 1985. p.129-49.

39. Tetzschner T, Sorensen M, Rasmussen OO, Lose G, Christiansen J. Pudenda nerve damage increase the risk of fecal incontinence in women with anal sphincter rupture after childbirth. Acta Obstet Gynecol Scand 1995;74:434 40 .

40. Thomas TM, Egan M, Meade TW. The prevalence and implications of faecal (and double) incontinence. Br J Surg 1985;72 Suppl:S141.

41. Thomas TM, Egan M, Walgrave A, Meade TW. The prevalence of faecal and double incontinence. Community Med 1984;6:216-20.

42. Vernava III AM, Longo WE, Daniel GL. Pudendal neuropathy and the importance of EMG evaluation of fecal incontinence. Dis Colon Rectum $1993 ; 36: 23-7$

43. Wexner SD, Salanga VD, Marchetti F, Corredor C, Jagelman DG. Neurophysiologic assessment of the anal sphincter. Dis Colon Rectum 1991;34:606-12. 